\title{
Formulation of Dynamics, Actuation, and Inversion of a Three-Dimensional Two-Link Rigid Body System
}

\author{
Hooshang Hemami \\ Department of Electrical Engineering \\ The Ohio State University \\ Columbus, Ohio 43210 \\ Behzad Dariush \\ Honda Research Institute \\ Suite 300 \\ Mountain View, California 94041 \\ Kamran Barin \\ College of Medicine \\ The Ohio State University \\ Columbus, Ohio 43210 \\ Received 19 July 2002; accepted 19 May 2005
}

\begin{abstract}
In this paper, three issues related to three-dimensional multilink rigid body systems are considered: dynamics, actuation, and inversion. Based on the Newton-Euler equations, a state space formulation of the dynamics is discussed that renders itself to inclusion of actuators, and allows systematic ways of stabilization and construction of inverse systems. The development here is relevant to robotic systems, biological modeling, humanoid studies, and collaborating man-machine systems. The recursive dynamic formulation involves a method for sequential measurement and estimation of joint forces and couples for an open chain system. The sequence can start from top downwards or from the ground upwards. Three-dimensional actuators that produce couples at the joints are included in the dynamics. Inverse methods that allow estimation of these couples from the kinematic trajectories and physical parameters of the system are developed. The formulation and derivations are carried out for a two-link system. Digital computer simulations of a tworigid body system are presented to demonstrate the feasibility and effectiveness of the methods. (c) 2005 Wiley Periodicals, Inc.
\end{abstract}

Journal of Robotic Systems 22(10), 519-534 (2005) (c) 2005 Wiley Periodicals, Inc.

Published online in Wiley InterScience (wnw.interscience.wiley.com). • DOI: 10.1002/rob.20083 


\section{INTRODUCTION}

Systems of interconnected rigid bodies for robotic and natural systems have been the subject of studies for many years. ${ }^{1-3}$ In developing the equations of motion of rigid body structures, the robotics community has especially focused on the problem of computational efficiency, with many of the most efficient algorithms based on a recursive Newton-Euler formulation. ${ }^{4}$ Indeed computational efficiency is important for the simulation and control of increasingly complex mechanisms of interconnected rigid bodies. However, equally important is developing formulations which are easy to implement and capable of addressing specific needs of not only robotic systems, but also natural systems for the purpose of simulating mechanisms of human motor control.

This paper addresses some of the basic issues related to dynamics and control of rigid body systems. This is especially true for natural systems, where the complexity of both body dynamics and motor control mechanisms have so far prevented widespread application of these models to physiological and clinical questions. Although the theoretical results of this paper are very general, the focus is to provide the analytical framework for systematic study of underlying neural control mechanisms that mediate walking and quiet-standing in human. For example, current conceptual models of the way humans maintain their upright stability are based on intuitive and empirical interpretation of experimental data. One such model is described by Nashner and his associates. ${ }^{5}$ Their approach is based on partitioning the movement space into discrete regions. Placement of the body in any of these regions generates a preprogrammed muscle activation pattern that attempts to restore upright stability. At least three different control strategies have been identified for maintaining postural stability in the sagittal plane: ankle strategy, hip strategy, and stepping strategy. ${ }^{6}$ Some investigators have expressed skepticism about the application of rigidbody dynamics to the strategy-oriented model of postural control. ${ }^{7}$ However, it is demonstrated that the inverted pendulum model of postural dynamics can be used to test the strategy-oriented hypotheses of control mechanisms. ${ }^{8}$

Addition of feedback elements that represent sensory and motor mechanisms to the threedimensional model described here can provide the framework to apply these models to clinical and physiological questions. This is not a trivial step. The feedback elements are most likely nonlinear, perhaps discrete, and in many cases highly simplified. None- theless, preliminary studies have demonstrated the feasibility of this approach. ${ }^{9,10}$ In many cases, the contribution of sensory modalities can be summarized in the form of measured angles and angular velocities. Similarly, the motor control mechanisms can be represented by actuators that generate joint torques.

The single rigid body case is considered first where free motion, constrained motion, and actuators are discussed. The two-link system is considered in Section 3. Stability and inverse plant are addressed in Sections 4 and 5. Simulations in Section 6, and discussion and conclusions in Section 7 conclude the paper.

\section{SINGLE RIGID BODY}

In this section the Newton-Euler equations are formulated. In the Newton-Euler formulation, the equations are not expressed in terms of independent variables and involve constraint forces, which must be eliminated in order to explicitly describe the input output relationships and arrive at closed-form equations. Arithmetic operations are needed to derive the closed-form dynamic equations and reduce the dimensionality of the system to a minimum. This represents a complex procedure which requires physical intuition. Systematic methods have previously been developed to project the dynamic equations of a larger dimensional state space to a closed form reduced state space. ${ }^{11}$ One contribution of this paper is to introduce systematic methods to recursively project the dynamic equations in order to eliminate joint reaction forces and redundant state variables.

Alternatives to the Newton-Euler formulation of rigid body dynamics are the Lagrangian and Kane's formulations, ${ }^{12}$ which describe the behavior of a dynamic system in terms of work and energy stored in the system rather than of forces and momenta of the individual members involved. The constraint forces involved in the system are automatically eliminated in the formulation and the closed-form dynamic equations can be derived systematically in any coordinate system. Closed form formulations based on Lagrangian formulation, Kane's formulations, and Newton-Euler with projections, ${ }_{11}^{11}$ have certain advantages in control; however with the exception of the latter, these methods have sometimes been criticized by those in the area of biomechanics and human movement science because most joint reaction forces are automatically eliminated early in the process of deriving dynamics equations. In addition, for mod- 
eling high degree of freedom systems, recursive methods are simpler to implement and considerably faster.

First, we present the dynamics of a single rigid body and introduce the notation. The formulation is then extended to two rigid bodies.

\subsection{Free Motion}

Before the state space of one rigid body and its formulation are presented, a transformation that converts cross product of vectors to a matrix transformation is presented. The introduction of a skew symmetric matrix allows the representation of vector cross products as a transformation. This is more convenient for computer simulation and calculations. To illustrate this issue, let a vector $R$ represent a position and a vector $F$, acting at the tip of vector $R$, represent a force. The torque of the force $F$ is described by the cross product of $R$ and $F$. The calculation of this torque can be carried out as a transformation:

$$
R \times F=\breve{\mathcal{R}} F .
$$

The skew symmetric $3 \times 3$ matrix $\breve{\mathcal{R}}$ is given in Appendix A.

With these preliminaries, consider a single rigid body. ${ }^{13}$ Let $\Theta$ and $\Omega$ be, respectively, the Euler angles and the angular velocity vector of the body, expressed in the body coordinate system (BCS)

$$
\begin{gathered}
\Theta=\left[\theta_{1}, \theta_{2}, \theta_{3}\right]^{\prime}, \\
\Omega=\left[\omega_{1}, \omega_{2}, \omega_{3}\right]^{\prime} .
\end{gathered}
$$

The sequence of angles above corresponds to roll, pitch, and yaw, respectively. Let $X$ and $V$ be the translational vectors of position and velocity of the center of gravity of the body in the inertial coordinate system (ICS). Let $\Lambda$ be a 3-vector of force acting at a point $c 1$ on the body whose coordinates are vector $R$ in BCS. This force could correspond to the vector of ground reaction forces. Let vector $\Lambda$ be specified in the ICS. In connection with vector $R$, we define the $3 \times 3$ matrix $\breve{\mathcal{R}}$. The effect of force $\Lambda$ is the cross product of $R$ and $\Lambda$. However, $\Lambda$ has to be transformed by a transformation $A^{\prime}(\Theta)$ so that both $R$ and $\Lambda$ are expressed in the BCS. The transformation matrix $A$, transforming a vector from $\mathrm{BCS}$ to
ICS, is given in Appendix A. The resulting torque from the action of $\Lambda$ is

$$
\breve{\mathcal{R}} A^{\prime} \Lambda \text {. }
$$

Let vectors of force $G$ and $H$ be, respectively, the gravity vector and the resultant vector of other forces acting on the body. The torque $N_{1}$ is the result of all couples and moment of all the remaining forces, except $\Lambda$, operating on the body. The diagonal moment of inertia matrix of the rigid body, along its principal axes, is given by $J$, where

$$
J=\operatorname{diag}\left[j_{1}, j_{2}, j_{3}\right] .
$$

The nonlinear vector $f(\Omega)$ is defined in Appendix A. An additional matrix $B(\Theta)$ defines the relation between $\Omega$ and $\dot{\Theta}$. Matrix $B$ is specified in Appendix A. With these definitions, the state space equations of motion of the single rigid body are

$$
\dot{\Theta}=B(\Theta) \Omega,
$$

$$
\begin{gathered}
J \dot{\Omega}=f(\Omega)+N_{1}+\breve{\mathcal{R}} A^{\prime} \Lambda, \\
\dot{X}=V,
\end{gathered}
$$

$$
m \dot{V}=G+H+\Lambda \text {. }
$$

The first two equations above describe the rotational motion of the rigid body. The last two equations describe the translation of the body in the ICS.

\subsection{Constrained Motion}

The inverted three-dimensional pendulum can be used to model the human torso, the human head or certain robots. The equations of the inverted pendulum can be systematically derived from the free motion equations above as shown below. We consider connection of the rigid body either to a stationary point or to a moving platform. Suppose the rigid body is permanently connected to a moving base or platform. The connection, is at the origin of the moving system. The connection point on the body is at a point $c 1$ with coordinates $R$. The vector of constraint (in this case contact with the ground) forces of con- 
straint is $\Lambda$. Let the motion of the platform origin be a known three-dimensional motion $X_{a}(t)$, described in the inertial coordinate system. These connection to the moving platform can be described by three holonomic constraints:

$$
\begin{gathered}
X+A R=X_{a}(t), \\
V+A \breve{\Omega} R=V_{a}(t), \\
\dot{V}+A(\breve{\Omega})^{2} R-A(\breve{R}) \dot{\Omega}=\dot{V}_{a}(t) .
\end{gathered}
$$

Sometimes it is desirable to reduce the system dimension to six. With the above definitions, the 12dimensional state space can be reduced, by projection, onto the space of $\Theta$ and $\Omega$. The forces of constraint are eliminated.

The reduced equations are

$$
\begin{gathered}
\dot{\Theta}=B(\Theta) \Omega, \\
{\left[J-m(\breve{\mathcal{R}})^{2}\right] \dot{\Omega}=f(\Omega)+N_{1}-\breve{\mathcal{R}} A^{\prime}\left(G+H-m \dot{V}_{a}\right)} \\
-m \breve{\mathcal{R}}(\breve{\mathcal{R}} \Omega) \Omega .
\end{gathered}
$$

The latter three of the above six equation can be further simplified: Let $J_{b}$ and $f_{b}$ be the moment of inertia matrix and the corresponding nonlinear function $f$ computed relative to a body coordinate system whose origin is at point $c 1$ :

$$
\begin{gathered}
J_{b}=J-m(\breve{\mathcal{R}})^{2}, \\
f_{b}=f-m \breve{\mathcal{R}}(\breve{\mathcal{R}} \Omega) \Omega .
\end{gathered}
$$

With the latter notation, the equations of the three-dimensional inverted pendulum result are

$$
\begin{gathered}
\dot{\Theta}=B(\Theta) \Omega, \\
J-b \dot{\Omega}=f_{b}(\Omega)+N_{1}-\breve{\mathcal{R}} A^{\prime}\left(G+H-m \dot{V}_{a}\right) .
\end{gathered}
$$

When it is desired to compute $\Lambda$, one can show that

$$
\Lambda=-G-H-m A(\breve{\Omega})^{2}(R)+m A \breve{R} \dot{\Omega}+m \dot{V}_{a}(t) .
$$

In the above equation, the force of constraint $\Lambda$ is given as a function of the state and the derivative of the state, i.e., $\dot{\Omega}$, rather than strictly as a function of the state and the input. An alternative method for arriving at the forces of constraint is to measure them by instrumenting the platform or by using force plates.

\subsection{Actuators}

The problem of introducing actuators in the single rigid body system is considered next. We restrict our study to motors for robots and muscles for natural systems. In order to relate this work to other formulations of rigid body dynamics, the input torques, moments, and couples will be specified in three coordinate systems: ICS, BCS and the $\Theta$ space. Motors apply input couples, such as $N$ in Eq. (1), to the rigid body. ${ }^{12}$ However, the motor is either connected to the ICS or to other rigid bodies. Therefore, it appears more expedient to formulate the the torque of the motor first in ICS and then transform it to BCS. The same is true for muscles and cablelike actuators. If the actuator is a pair of rockets, directly mounted on the rigid body, then it is more convenient to formulate the torque produced directly in BCS. We first describe a physical situation that arises often in robotics. ${ }^{14}$ Suppose the motion of the inverted pendulum is further restricted by rotation about a stationary shaft in the ground. Suppose the rotation of the rigid body is brought about by a motor with torque of magnitude $u_{1}$. It is assumed there is no friction. Let the direction of the shaft in ICS be described by a unit vector $\alpha$. The torque acting on the pendulum, expressed in BCS is $A^{\prime} \alpha u_{1}$. Therefore, in the BCS

$$
N=A^{\prime} \alpha u_{1} .
$$

Sometimes, the equations of the pendulum are written in Lagrangian form where the state space is composed of state variables $\Theta$ and $\dot{\Theta}$. In order to transform $N$ to the latter state space, the principle of ritual work is invoked. The incremental work $d w$, delivered to the inverted pendulum is expressed in the $\Theta$ space as 


$$
d w=M^{\prime} d \Theta .
$$

The instantaneous power

$$
p=\partial w / \partial t
$$

delivered to the pendulum, is

$$
p=M^{\prime} \dot{\Theta} .
$$

The power delivered to the inverted pendulum, as described in BCS is

$$
p=N^{\prime} \Omega .
$$

It follows from Eqs. (6) and (7) that

$$
N=B^{\prime} M
$$

The vector $M$ is the torque of the shaft in the Lagrangian equations of motion.

Let the actuators collectively produce the couple vector $U$, expressed in the ICS system. Physically, three pairs of musclelike actuators could also produce the couple $U$ in order to control the human trunk motion. Following the same argument as above, it follows that

$$
N=A^{\prime} U
$$

and

$$
N=B^{\prime} M,
$$

as before where the vector $M$ appears in the Lagrangian form of the equations of motion.

The torque of muscles usually involves knowing the vector force of the muscle, its moment arm, its length, and its velocity (contraction or extension rate). These computations are easier to do in the ICS.

Suppose the vector of muscle force is $F$, and the muscle is connected to a point of the rigid body with coordinates $R$ in the body coordinate system. The torque of this muscle in the body coordinate BCS is

$$
N=\breve{\mathcal{R}} F
$$

Let the same torque be represented by $u$ in ICS. It follows that

$$
u=A \breve{\mathcal{R}} F
$$

To compute $u$ directly in ICS, we first transform both vectors $R$ and $F$ and then perform the cross product operation

$$
u=(\breve{A R}) A F .
$$

An interesting theoretical result, which can also be independently derived, is the following. By equating $u$ in Eqs. (10) and (11), and, assuming that $F$ is arbitrary, the following identity results:

$$
(\breve{A R})=A \breve{\mathcal{R}} A^{\prime},
$$

which is true for any $R$ and any orthonormal matrix A.

\section{TWO RIGID BODIES}

\subsection{Free Body Equations}

We consider a system of two rigid bodies. Let us assume body 1 is connected to a moving platform as described in 1 . Suppose a point $c 2$ of body 2 is connected to a point $d 1$ of body 1 . The two rigid body system is depicted in Figure 1. For convenience, subscripts 1 and 2 will designate the bodies 1 and 2 . The point $d 1$ has coordinates $S_{1}$ in BCS1, and point $c 2$ has coordinates $R_{2}$ in BCS2. Let the vector of holonomic force of contact, acting on body 2 , be $\Lambda_{2}$, expressed in the ICS. The force acting on body 1 is $-\Lambda_{2}$. Equations (1) of body 1 will be modified in order to integrate the effect of the interaction force and the interaction couple with body 2. For completeness and in order to make the recursion complete, the motion of point $c 2$ will be designated as $X_{b}(t)$. For convenience equations of body $i(i=1,2)$ are written with subscripts $i$. The equations for body 1 are

$$
\begin{gathered}
\dot{\Theta}_{1}=B_{1}\left(\Theta_{1}\right) \Omega_{1} \\
J_{1} \dot{\Omega}_{1}=f_{1}\left(\Omega_{1}\right)+N_{01}-N_{21}+\breve{R}_{1}\left(A_{1}\right)^{\prime}\left(\Lambda_{1}\right)-\breve{S}_{1}\left(A_{1}\right)^{\prime} \Lambda_{2}
\end{gathered}
$$

$$
\dot{X}_{1}=V_{1}
$$




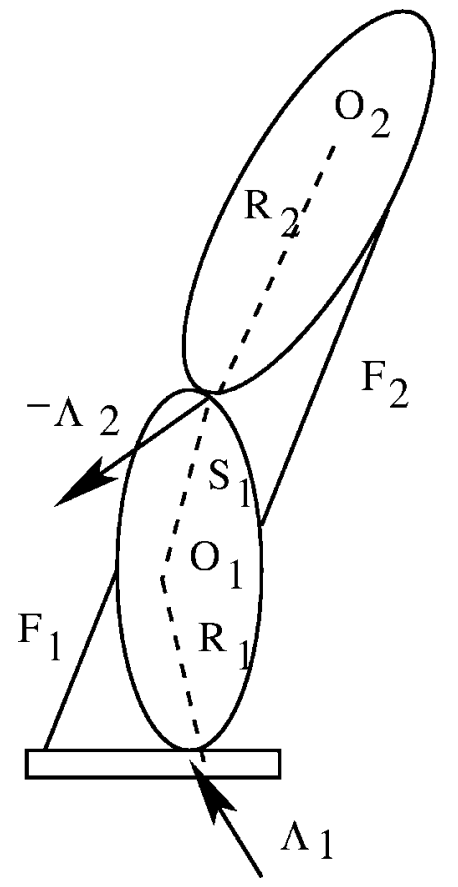

Figure 1. The two-link rigid body system joined at a point.

$$
m_{1} \dot{V}_{1}=G_{1}+H_{1}+\Lambda_{1}-\Lambda_{2}
$$

where $N_{01}$ is the torque of the moving platformbased actuator system and $N_{21}$ is the effect on body 1 of the actuators, situated between the two bodies, and expressed in BCS1. The four free body equations for body 2 are

$$
\begin{gathered}
\dot{\Theta}_{2}=B_{2}\left(\Theta_{2}\right) \Omega_{2}, \\
J_{2} \dot{\Omega}_{2}=f_{2}\left(\Omega_{2}\right)+N_{12}+\breve{R}_{2} A_{2}^{\prime} \Lambda_{2}, \\
\dot{X}_{2}=V_{2} \\
m_{2} \dot{V}_{2}=G_{2}+H_{2}+\Lambda_{2} .
\end{gathered}
$$

The couple $N_{12}$ is the effect of the actuation between bodies 1 and 2, expressed in BCS2.

\subsection{Constraints}

Following the same procedure as for the single rigid body, the motion of point $\mathrm{d} 1$ of body 1 is

$$
X_{b}(t)=X_{1}+A_{1} S_{1}
$$

The connection constraints between bodies 1 and 2 are

$$
\begin{gathered}
X_{2}+A_{2} R_{2}=X_{b}(t) \\
V_{2}+A_{2} \breve{\Omega}_{2} R_{2}=V_{b}(t) \\
\dot{V}_{2}+A_{2}\left(\breve{\Omega}_{2}\right)^{2} R_{2}-A_{2}\left(\breve{R}_{2}\right) \dot{\Omega}_{2}=\dot{V}_{b}(t) .
\end{gathered}
$$

The connection constraints, namely, Eqs. (2), (20), and (21) can be used to compute the forces of constraint or reduce the dimension of the system by projecting the state space of the free body system onto the state space of the double inverted pendulum: the space of the angles and angular velocities of the two bodies

$$
\Theta_{1}=\Theta_{1}
$$

$$
\Omega_{1}=\Omega_{1}
$$$$
X_{1}=-A_{1} R_{1}
$$

$$
V_{1}=A_{1} \breve{R}_{1} \Omega_{1}
$$

$$
\Theta_{2}=\Theta_{2}
$$

$$
\Omega_{2}=\Omega_{2}
$$

$$
X_{2}=A_{1}\left(S_{1}-R_{1}\right)-A_{2} R_{2}
$$

$$
V_{2}=A_{1}\left(S_{1} \breve{-} R_{1}\right) \Omega_{1}+A_{2} \breve{R}_{2} \Omega_{2} .
$$

These projection equations are used in stability, as shown later, and in computation of the forces of con- 
straint in the reduced state space of the double inverted pendulum.

\subsection{Double Inverted Pendulum}

A double inverted pendulum can be used to model natural systems or robotic systems. Head and neck, head and trunk, or head and neck systems can be represented by double pendulums. Robots with two links, and three degree of freedom at the joints can be similarly modeled. One can, systematically, reduce the above equations of motion to those of a double inverted pendulum. For this purpose, the forces $\Lambda_{1}$ and $\Lambda_{2}$, as well as the translational position and velocity variables are eliminated from the equations of motion and the constraints by the above projection equations. The details of the derivation are not presented here. These equations of the double pendulum are symbolically, written as

$$
\mathcal{J} \dot{\Omega}=\mathcal{D}+\mathcal{F}+\mathcal{G}+\mathcal{H}+\mathcal{N}
$$

where $\mathcal{D}$ is the centrifugal term, $\mathcal{F}$ is the vector of nonlinear terms: $f_{1}$ and $f_{2}, \mathcal{G}$ is the vector of gravity, $\mathcal{H}$ is the effect of the two resultant vectors of force on bodies 1 and 2. Finally $\mathcal{N}$ describes the total torques acting on the two bodies. The precise formulas for all six six symbols are given in Appendix B.

\subsubsection{Additional Constraints}

Suppose a rigid cable with fixed length connects an arbitrary point $e_{1}$ of body 1 to an arbitrary point $e_{2}$ on body 2 . The cable is assumed to be in a straight line. Let vectors $E_{1}$ and $E_{2}$, respectively, be the coordinates of these points in bodies 1 and 2. The holonomic constraint resulting from this connection is that the length of the cable remains constant in the ICS coordinate system. The vector $L$, extending from point $e_{1}$ to point $e_{2}$ is expressed as

$$
e_{1} e_{2}=\left(X_{2}+A_{2} E_{2}\right)-\left(X_{1}+A_{1} E_{1}\right) .
$$

The holonomic constraint, due to the cable, is expressed as

$$
L^{\prime} L=\text { const }
$$

Let the force that the cable applies to body 1 be $\Gamma$ in ICS. The effect of this force on body 1 is

$$
N_{12}=\breve{E}_{1} A_{1}^{\prime} \Gamma \text {. }
$$

The effect of this force on body 2 is

$$
N_{21}=\breve{E}_{2}\left(A_{2}\right)^{\prime} \Gamma \text {. }
$$

From the equations of the two body system, i.e., Eqs. (13)-(20) and the constraint equation (24), the force $\Gamma$ can be computed. An example of such a constraint is when one voluntarily stiffens muscles such that one limb does not move very easily relative to another one. One can similarly represent chains and gears, but this issue is not pursued here.

\subsection{Actuators}

We consider a passive actuator first. Suppose the cable is replaced by a passive actuator made of one linear spring with constant $k$ and a linear shock absorber with constant $b$. Ligaments in natural systems can be represented in this way. Suppose the length and velocity of the spring between the two points of contact on the two bodies are, respectively, $l$ and $i$-easily computable in ICS. The unit vector in the direction $e_{1} e_{2}$, expressed in ICS, is

$$
\beta=L /(\|L\|),
$$

where the notation $\|$.$\| signifies length. With these$ definitions

$$
\Gamma=\left[k\left(l-l_{0}\right)+b i\right] \beta .
$$

Now suppose the spring and dash-pot is replaced by an active actuator such as a muscle. In this case the equation for $\Gamma$ would also involve, in addition to its length and velocity, the internal states of the actuator, if any, and also its neural or command inputs. Furthermore, when the moment arm of the muscle is the same for both rigid bodies connected by the muscle, the resulting torque acting on one rigid body is of the same magnitude as the torque acting on the second body but of opposite sign. Physically this means the muscle is parallel with the line $\mathrm{O}_{1} \mathrm{O}_{2}$ connecting the centers of gravity of the two bodies (see Figure 1). 


\section{STABILITY OF THE DOUBLE INVERTED PENDULUM}

In this section, first the philosophy of the stabilizing control, and the background of the control strategy are presented. The synthesis and the implementation follow.

\subsection{Philosophy}

The philosophical point of view is to carry out the stability issue in three steps:

Step 1. Stabilize the free body system, namely the system of equations (13)-(20), and construct a Lyapunov function to estimate the region of stability of the system.

Step 2. Derive parametric equations that project the state space onto the reduced state space of the double inverted pendulum.

Step 3. Project the state space, the controls and the Lyapunov function onto the reduced state space.

For simplicity, we assume that additional nonlinear feedback is used to compensate for gravity, or, alternatively, assume that gravity is equal to zero. We assume a set of actuator couples $N$ are available that, similar to the action of rockets, produce torques on each body separately:

$$
N=\left[N_{1}^{\prime}, N_{2}^{\prime}\right]^{\prime} .
$$

We propose nonlinear position and linear velocity feedback here. ${ }^{15,16}$ as follows: Let $K r, L r, K t$, and $L t$ be four $3 \times 3$ positive definite matrices. The feedback needed for control of the rotational motion is given by

$$
\begin{aligned}
& N_{1}=-B_{1}^{\prime} K r_{1}\left(\Theta_{1}\right)-L r_{1} \Omega_{1}, \\
& N_{2}=-B_{2}^{\prime} K r_{2}\left(\Theta_{2}\right)-L r_{2} \Omega_{2} .
\end{aligned}
$$

A similar control structure is proposed for the translational motion:

$$
H_{1}=-K t_{1}\left(X_{1}-X 0_{1}\right)-L t_{1} V_{1}-G_{1}
$$

$$
H_{2}=-K t_{2}\left(X_{2}-X 0_{2}\right)-L t_{2} V_{2}-G_{2},
$$

where $G$ is the gravity vector. For Lyapunov function, the sum of the kinetic, elastic and potential energies are used. The translational energy $v_{t}$ and the rotational energy $v_{r}$ for each of the two rigid bodies, are, respectively,

$$
v_{t}=0.5 V^{\prime} M V+0.5\left(X-X_{0}\right)^{\prime} K t\left(X-X_{0}\right),
$$

$$
v_{r}=0.5 \Omega^{\prime} J \Omega+0.5(\Theta)^{\prime} \operatorname{Kr}(\Theta) .
$$

The overall Lyapunov function $v$ for the two rigid body system is defined by

$$
v=v_{t} 1+v_{r} 1+v_{r} 2+v_{t} 2
$$

It can be shown that candidate $\mathrm{v}$ satisfies the necessary conditions so that $v$ is a Lyapunov function. It can also be shown that the derivative of $v$ with respect to time is nonpositive, ${ }^{15}$ and therefore $v$ is a Lyapunov function for the system, and that the system is asymptotically stable.

Remark 1. In the present strategy, the gravity is compensated by nonlinear feedback. This is the reason for the absence of the potential energy as a component of the Lyapunov function.

Naturally, the choice of the gains in the above general feedback structures depends on the gravity constant, the load, and the complexity of the system. In the two link system, considered here, the values for the stabilizing feedback gain matrices of body 1 depend on how heavy body 2 is, the gravity constant, and what other loads act on body 2 .

\subsection{Controller Design}

The controller that stabilizes the double inverted pendulum, is designed in four steps.

1. Four vector actuators are considered. These are the two torque vectors and the two force vectors acting, respectively, on the rotational subsystems and the translational sub-systems of the two bodies.

2 . The eight $3 \times 3$ gain matrices, for stability are specified.

3. The 24 state variables of the two bodies, appearing in these controller equations are projected onto the 12 state variables: the space of the angles and angular velocities of the two bodies, as previously explained. 
4. The same transformation, i.e., Eq. (22) is applied to the Lyapunov function in order to define the Lyapunov function for the 12 dimensional reduced state space of the double inverted pendulum, and its derivative with respect to time.

Let the projection of $\left[\mathrm{H}_{1}, \mathrm{H}_{2}\right]$ onto the double inverted pendulum state space be designated by $\mathcal{H}_{p}$. Let the projection of $\left[N_{1}, N_{2}\right]$ be $\mathcal{N}_{p}$.

The final step in the control is to implement $\mathcal{H}_{p}$ and $\mathcal{N}_{p}$ with six actuators - three for each pendulum.

\section{INVERSE PLANT}

The need for the inverse of a robotic system or a human model arises in many situations ${ }^{17}$ including diagnostics. ${ }^{18}$ Broadly speaking, the input to the inverse system is the desired state trajectories. The output of the inverse plant is the required torques, forces, etc., that shape the actual system's state trajectories.

In point to point movements, the input couples to the system must be programmed. In diagnostics, athletic assessment and rehabilitation, it is desirable to estimate joint torques and couples. It is preferable that the estimation be noninvasive. One way to do this is to ask the subject to engaged in certain prespecified maneuvers or exercises, 10 and from measurement of reaction forces and the observed trajectories of motion, estimate joint forces, and joint torques. The alternative method is to tune a computer model to the physical parameters and attributes of the subject, and let the computer model perform the same motion for arriving at the joint forces and torques as estimates of those of the subject. Both methods require good inverse plants.

In another class of problems, there are no external inputs. The subject or the system is disturbed, and the healthy maneuver is to return to the undisturbed equilibrium point. The objective here is to estimate the internal stabilizing torques and couples from the measurement of trajectories. A convenient mechanism for this purpose is to subject the biped or robot system to motions induced by rotational or translational motion of the platform. As Eq. (5) shows, the effect of translation of the platform is equivalent to application of a force to the center of gravity of the biped equal to the mass of the first link times the acceleration of the base. The platform motion, is one of three types: random motion, periodic motion, or transient pulselike motion. The measured response of the subject provides information about the state of

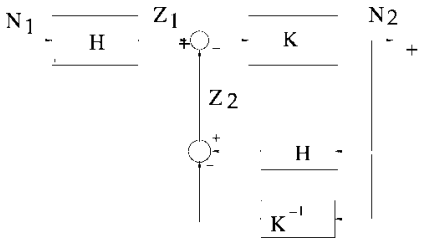

Figure 2. The two-link rigid body system $\mathrm{H}$ and its inverse.

health, etc. The inverse plant concept helps to estimate the internal torques, forces, and couples (ref. 19, Chap. 13).

The methodology here allows for

1. Systematic development of the inverse,

2. systematic ways of stabilizing the inverse, and

3 . assessing the performance of the inverse.

The main requirement is that the system is subjected to periodic inputs or inputs of finite energy, i.e., signals that eventually go to zero.

Suppose the two-link inverted pendulum is characterized by an operator $H$. The inverse plant, as shown in Figure 2 is to be constructed. There are different approaches to the construction of the inverse plant. We use a methodology developed by Zames ${ }^{20}$ and George. ${ }^{21}$ The inverse system requires a replica of the system to be inverted in the feedback path, an operator $K$ in the forward path, and the operator $K^{-1}$ in parallel with $H$ of the inverse as shown in Figure 2. More detail about the structure, stability and application of the inverse to a one-link rigid body system is provided in ref. 22. The input $N_{1}$ to system $H$ consists of two couples, abstractly designated as $N_{1}$. The output of system $H$ is assumed to be its whole state, designated as $Z_{1}$. The latter assumption allows one to avoid difficulties associated with zero dynamics, ${ }^{23}$ and general inverses. ${ }^{24}$ One advantage of the above inverse is that its stability can be guaranteed.

When the original system $H$ is bounded inputbounded output (i.e., BIBO) stable, ${ }^{25}$ the inverse is also stable in the same sense. Alternatively, one can show that the inverse system is globally stable for the range of states for which the existence criterion of Lipschitz condition ${ }^{26}$ holds. The proof is straightforward, particularly when $K$ is a positive definite and large operator such that the inverse $K^{-1}$ can be ignored. The latter case, i.e., with $\mathrm{K}^{-1}$ set equal to zero can also be studied within the realm of systems with large gain. ${ }^{27}$ 

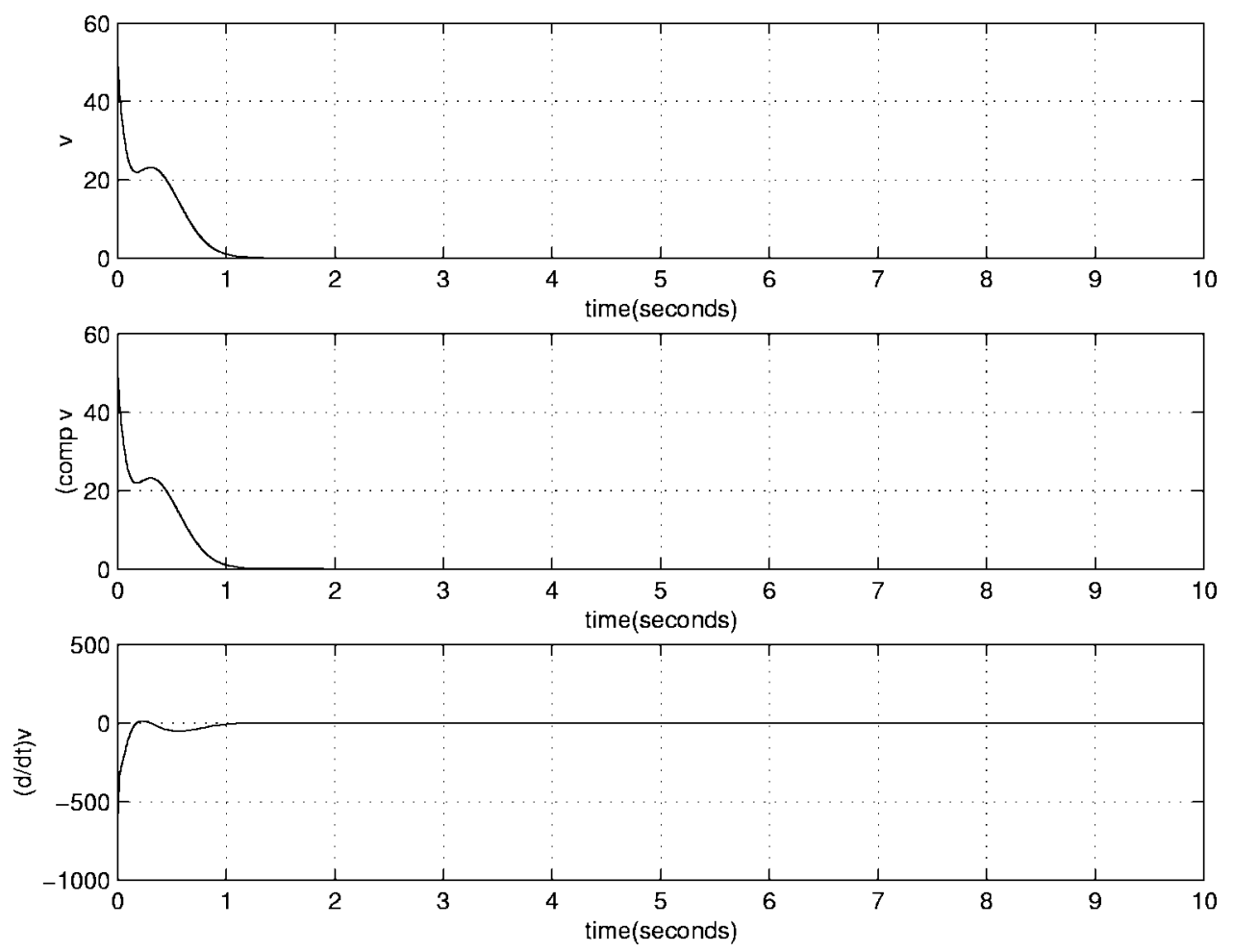

Figure 3. Two calculations of the Lyapunov function and its derivative with respect to time versus time.

Suppose the stable three-dimensional double inverted pendulum is to be inverted when the vector of periodic input couple is applied to link two. We consider an inverse with $\mathrm{K}^{-1}$ set equal to zero. The output of the double inverted pendulum is the six position and velocity states of link two, and the operator $K$ is taken to be a diagonal $6 \times 6$ matrix. A thorough consideration of inverses in general, the domain of their stability, and compromises of accuracy versus global stability is beyond the scope of this paper, and merits further study. We present some computer simulations in order to focus attention to this class of inverses.

\section{SIMULATION RESULTS AND DEMONSTRATIONS}

\subsection{Transient Response}

In this example, the two-link double inverted pendulum system is disturbed from the vertical equilibrium state. Its return to the equilibrium point is simulated. The double inverted pendulum is represented in Eq. (23). The detailed equations are listed in Appendix B. Stability is achieved by designing nonlinear position and velocity feedback according to Eqs. (26) and (27) for both links, and then projecting these controls onto the 12-dimensional reduced state space. The numerical parameters and initial conditions are given in Appendix C. Figure 3 shows two computations of the Lyapunov function and the derivative of the Lyapunov function with respect to time for 10 seconds. Figure 4 shows the angular positions, i.e., $\Theta_{1}$ and angular velocities $\Omega_{1}$ as functions of time. Figure 5 shows the same for trajectories of body 2 .

\subsection{Periodic Response}

The stable double inverted pendulum of the previous simulation was subjected to a periodic couple input. The input couple is active only on body two. As shown in Figure 6, the components of the couple acting on body 1 are zero, and the only nonzero component of the couple on body two is along the first principal axis of the body. The corresponding trajectories for $\Theta_{1}$ and $\Omega_{1}$ are shown in Figure 7. The trajectories for body two are shown in Figure 8. 

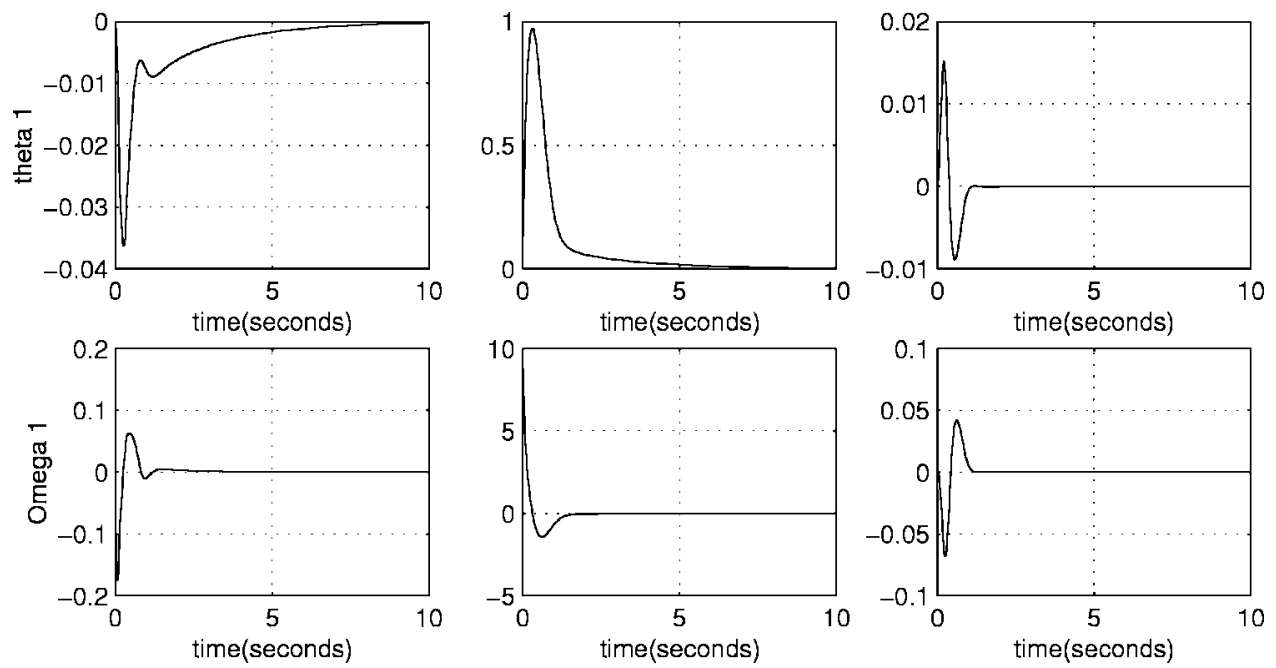

Figure 4. The trajectories of body 1 with respect to time.

The latter state trajectories are input to the inverse system of Figure 2. The corresponding state trajectories of the inverse, and the estimated input couple - as the output of the inverse system are, respectively, plotted in Figures 9-11. The estimates of the couple, and the state trajectories of the inverse compare favorably with those of double pendulum.

\section{DISCUSSION AND CONCLUSIONS}

The main contribution of this paper is in providing a systematic methodology for deriving the equations of motion for interconnected rigid body systems, in developing stability mechanisms and constructing Lyapunov functions, and in the design of inverses that are stable, and whose performance can be assessed. The formulation is applicable to natural as well as man made systems. The method yields very efficiently to construction, stability, and performance assessment of the inverse plants. Specifically the model is applicable to the study of human postural stability due to perturbations.

The approach can contribute to clinical tests of postural control by identifying outputs of the system
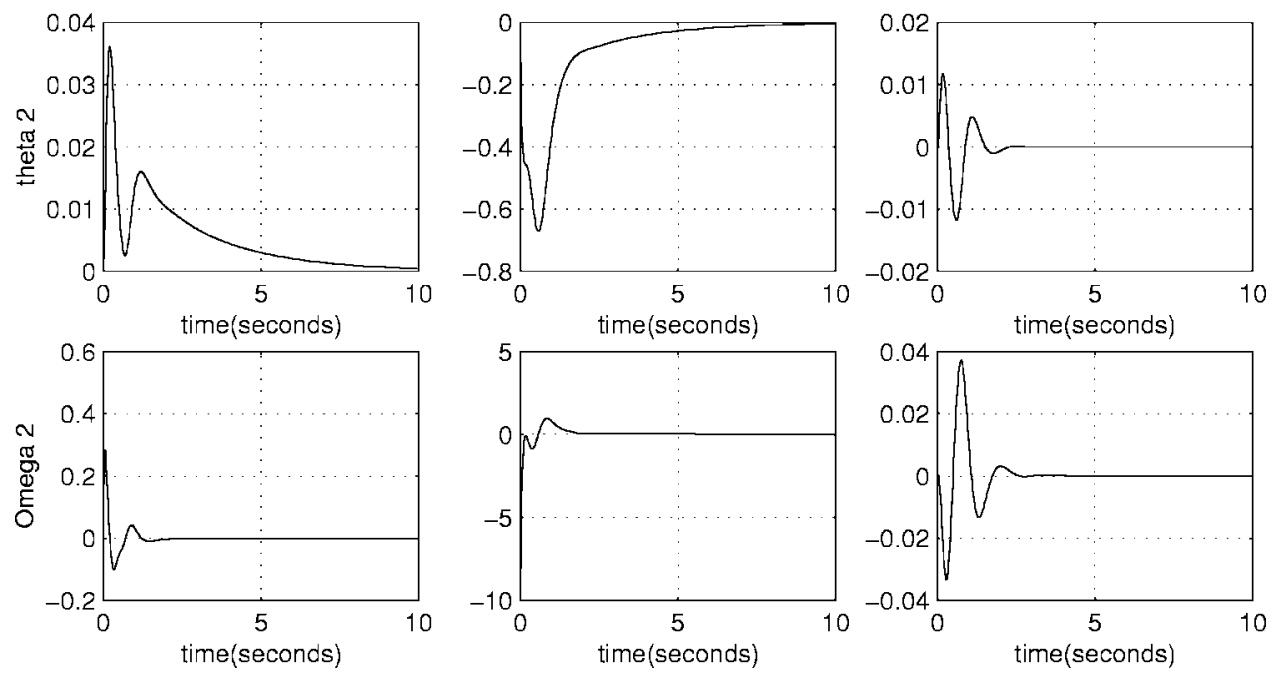

Figure 5. The trajectories of body 2 with respect to time. 

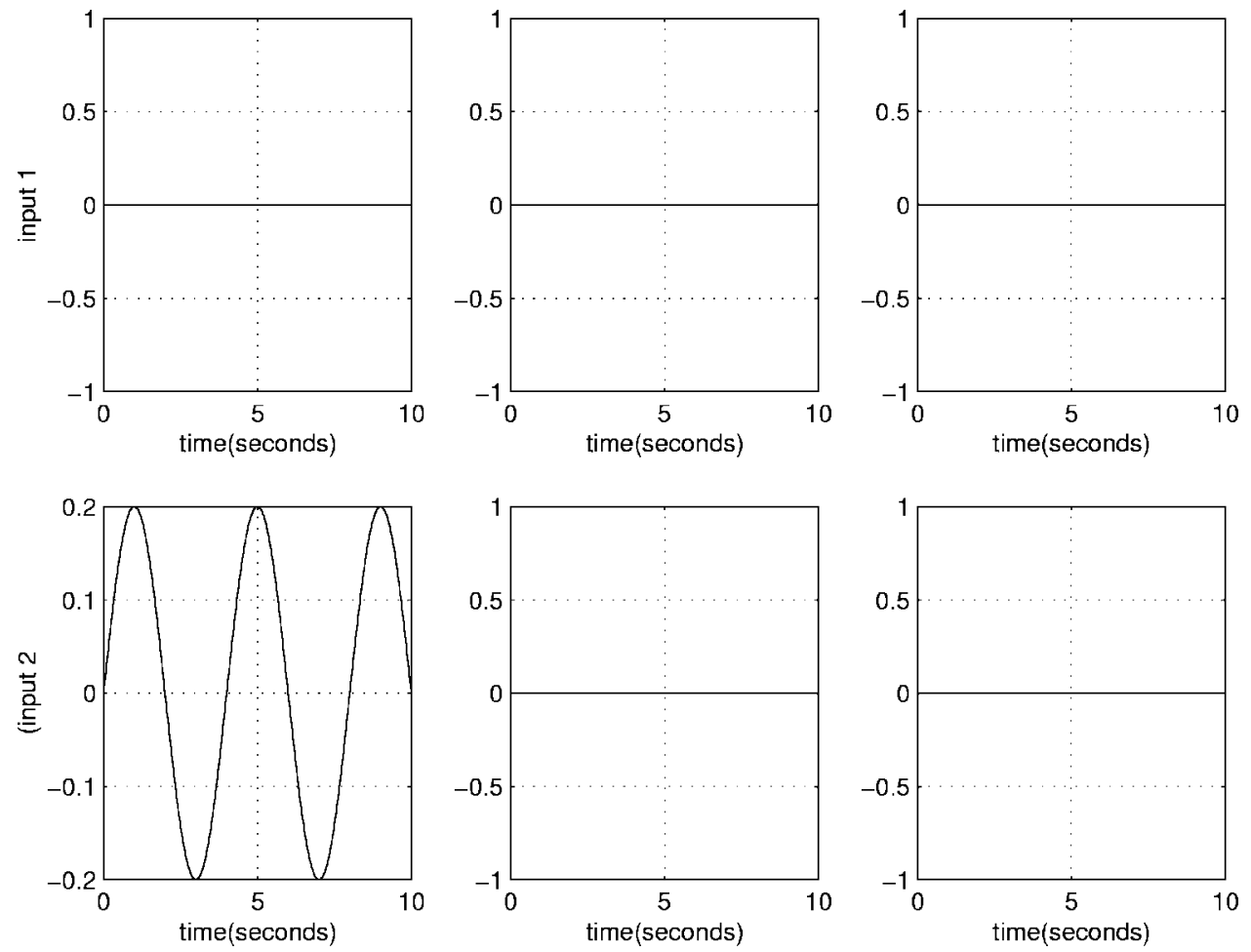

Figure 6. The periodic input couple to bodies one and two.

that are most sensitive to body's feedback mechanisms. Currently, the measured variables that are used to characterize postural stability, e.g., body center of mass or foot center of pressure, are selected mostly on intuitive grounds. It is not clear if some of the sensory modalities are observable through these outputs and if so, how sensitive those outputs are to changes in the status of the sensory signals. The proposed model provides the framework for formal analysis of sensitivity and observability for different feedback mechanisms of the body that represent various sensory and motor mechanisms.
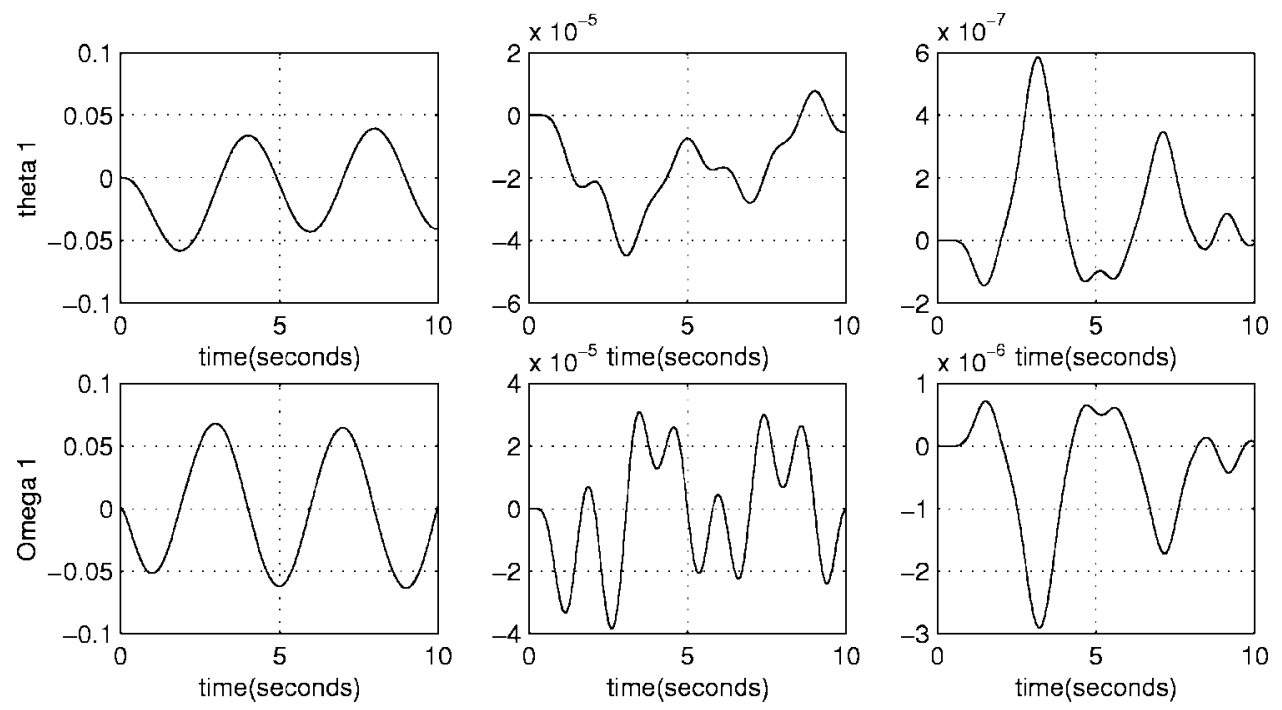

Figure 7. The trajectories of body 1 with respect to time for the periodic input couple of Figure 6. 

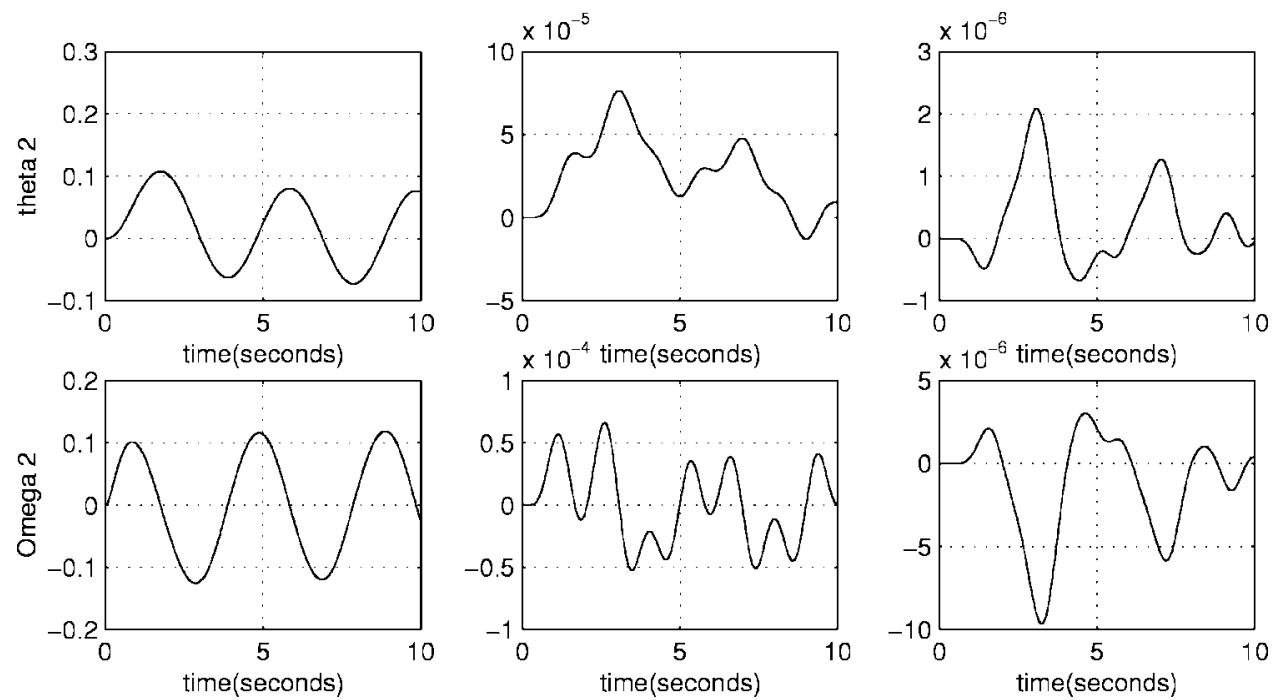

Figure 8. The trajectories of body 2 with respect to time for the periodic input couple of Figure 6.

Another application is in the area of determining perturbations that optimize the sensitivity of the output to particular feedback mechanisms of the body. Clinical tests of posture often use transient step responses of the system. Some attempt is made to modulate contributions of vision or proprioception by moving the visual surround and the support surfaces. There is no guarantee that these stimuli are optimal for detecting abnormalities in the postural control mechanisms. The modeling method provides a systematic way to find perturbations that maximize the sensitivity of the response variables to changes in specific sets of feedback mechanisms. As a result, test protocols can be devised that are more sensitive to abnormalities in postural control mechanisms associated with those feedback mechanisms.

The methodology has applications beyond study of human postural balance. Recursive procedures can be added that are directly applicable to computa-
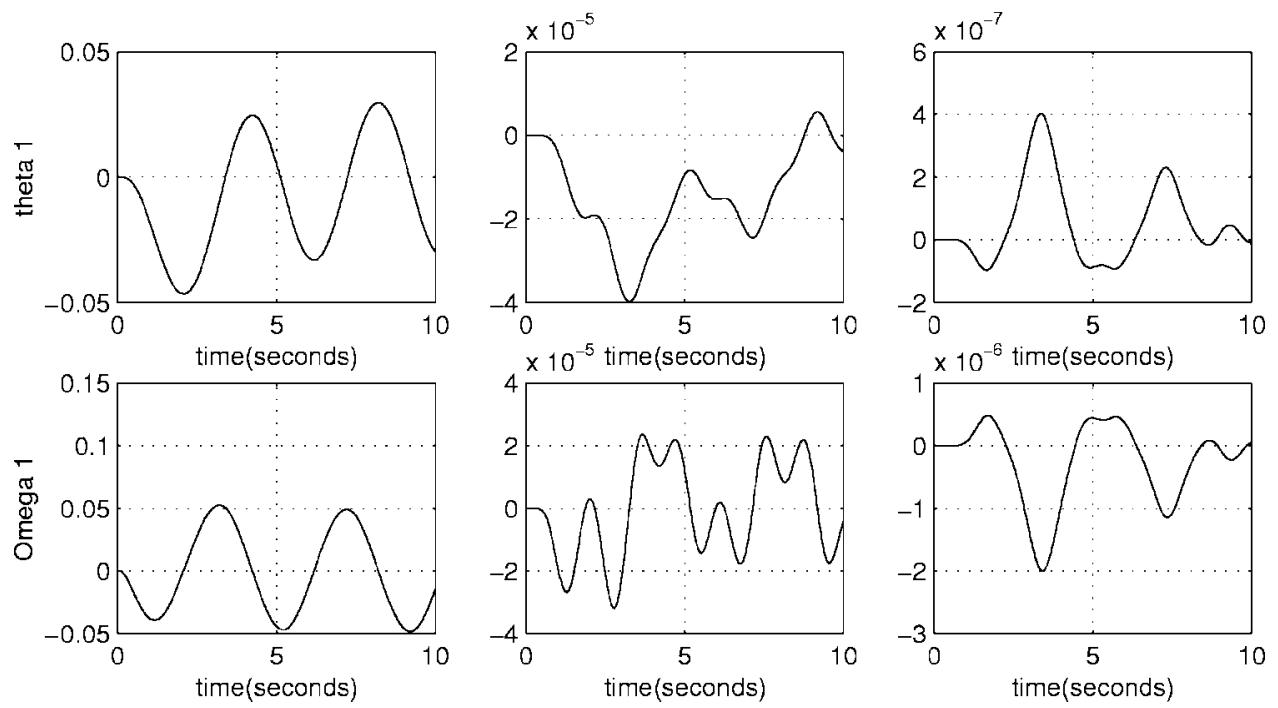

Figure 9. The trajectories of body 1 in the inverse system with respect to time. 

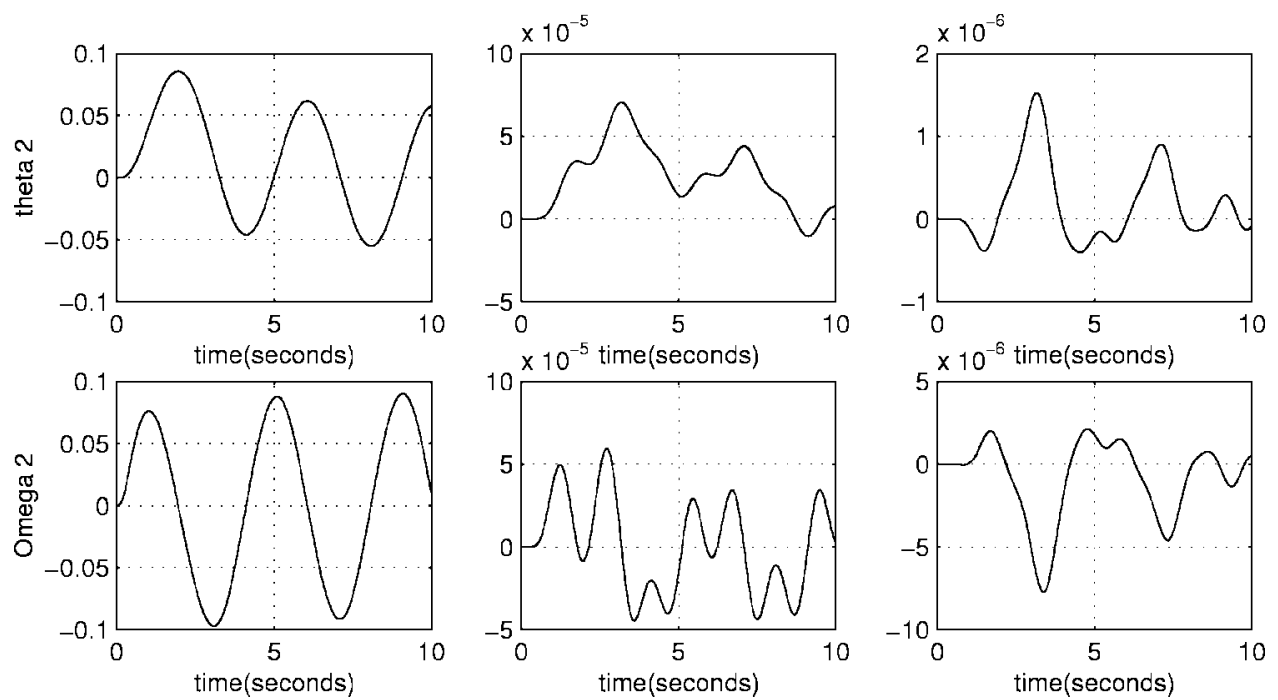

Figure 10. The trajectories of body 2 in the inverse system with respect to time.

tional methods for analysis, and synthesis of control strategies for exoskeletons and human augmentation devices.

$$
\breve{\mathcal{R}}=\left[\begin{array}{ccc}
0 & -r_{3} & r_{2} \\
r_{3} & 0 & -r_{1} \\
-r_{2} & r_{1} & 0
\end{array}\right] .
$$

\section{APPENDIX A: ONE RIGID BODY}

Let vector $R$ have components $r_{1}, r_{2}$, and $r_{3}$. The skew symmetric matrix $\breve{\mathcal{R}}$ is defined as
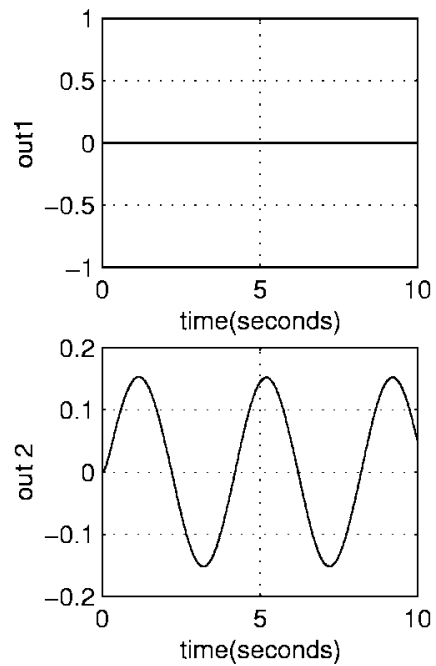

The nonlinear vector $f(\Omega)$ is given by

$f(\Omega)=\left[-\omega_{2} \omega_{3}\left(j_{3}-j_{2}\right),-\omega_{3} \omega_{1}\left(j_{1}-j_{3}\right),-\omega_{1} \omega_{2}\left(j_{1}-j_{2}\right)\right]^{\prime}$.

Figure 11. The estimated input couple as output of the inverse system. 
The matrices $A(\Theta)$, and $B(\Theta)$ that appear in the formulation of single rigid bodies are given below.

Let $A_{1}\left(\theta_{1}\right), A_{2}\left(\theta_{2}\right)$ and $A_{3}\left(\theta_{3}\right)$ be defined by

$$
\begin{aligned}
& A_{1}\left(\theta_{1}\right)=\left[\begin{array}{ccc}
1 & 0 & 0 \\
0 & \cos \theta_{1} & -\sin \theta_{1} \\
0 & \sin \theta_{1} & \cos \theta_{1}
\end{array}\right], \\
& A_{2}\left(\theta_{2}\right)=\left[\begin{array}{ccc}
\cos \theta_{2} & 0 & \sin \theta_{2} \\
0 & 1 & 0 \\
-\sin \theta_{2} & 0 & \cos \theta_{2}
\end{array}\right], \\
& A_{3}\left(\theta_{3}\right)=\left[\begin{array}{ccc}
\cos \theta_{3} & -\sin \theta_{3} & 0 \\
\sin \theta_{3} & \cos \theta_{3} & 0 \\
0 & 0 & 1
\end{array}\right] .
\end{aligned}
$$

Now $A(\Theta)$ can be defined

$$
A(\Theta)=A_{1}\left(\theta_{1}\right) A_{2}\left(\theta_{2}\right) A_{3}\left(\theta_{3}\right) .
$$

The orthonormal matrix $A$ transforms vectors from the BCS to ICS. The matrix $A^{\prime}$ which is the transpose and the inverse of $A$, transforms the vectors from the ICS to BCS.

The matrix $B(\Theta)$ is given by

$$
B(\Theta)=\left[\begin{array}{ccc}
\frac{\cos \theta_{3}}{\cos \theta_{2}} & \frac{-\sin \theta_{3}}{\cos \theta_{2}} & 0 \\
\sin \theta_{3} & \cos \theta_{3} & 0 \\
\frac{-\sin \theta_{2} \cos \theta_{3}}{\cos \theta_{2}} & \frac{\sin \theta_{2} \sin \theta_{3}}{\cos \theta_{2}} & 1
\end{array}\right] .
$$

\section{APPENDIX B: TWO RIGID BODY SYSTEM}

The six symbols, defining the equations of the two body system (23) are defined here. The matrix $\mathcal{J}$ is the $6 \times 6$ symmetric matrix whose four $3 \times 3$ elements are

$$
\left[\begin{array}{cc}
J b_{1}+\left(S_{1} \breve{-} R_{1}\right)^{\prime} m_{2}\left(S_{1}-R_{1}\right) & \left(S_{1} \breve{-} R_{1}\right) A_{1} m_{2} A_{2} \breve{R}_{2} \\
\breve{R}_{2}\left(A_{2}\right)^{\prime} m_{2} A_{1}\left(R_{1}-S_{1}\right) & J b_{2}
\end{array}\right] .
$$

The centrifugal term $\mathcal{D}$ is given by

$$
D=\left[\begin{array}{c}
-m_{2}\left(S_{1}-R_{1}\right)\left(\breve{\Omega}_{1}\right)^{2}\left(S_{1}-R_{1}\right)+A_{1}^{\prime} A_{2}\left[\left(\Omega_{2}\right)^{2} R_{2}\right] \\
-m 2 \breve{R}_{2} A_{2}^{\prime} A_{1}\left(\breve{\Omega}_{1}\right)^{2}\left(S_{1}-R_{1}\right)
\end{array}\right] .
$$

The vector $\mathcal{F}$ is given by the two nonlinear terms appearing in Eqs. (14) and (18):

$$
\mathcal{F}=\left[f_{1}^{\prime}, f_{2}^{\prime}\right]^{\prime} .
$$

The effect of the applied forces $\mathcal{H}$ and the gravity vector $\mathcal{G}$ are identical and are given by

$$
\mathcal{G}=\left[-\breve{R}_{1} A_{1}^{\prime}\left(G_{1}\right)+\left(S_{1}-{ }^{-} R_{1}\right) A_{1}^{\prime}\left(G_{2}\right),-\breve{R}_{2} A_{2}^{\prime}\left(G_{2}\right)\right]^{\prime}
$$

and

$$
\begin{aligned}
\mathcal{H}= & {\left[-\breve{R}_{1} A_{1}^{\prime}\left(H_{1}+m_{1} \dot{V}_{a}\right)+\left(S_{1}-\breve{-} R_{1}\right) A_{1}^{\prime}\left(H_{2}\right),\right.} \\
& \left.-\breve{R}_{2} A_{2}^{\prime}\left(H_{2}\right)\right]^{\prime},
\end{aligned}
$$

where it is assumed that the platform has a prescribed motion. The input torque vector $\mathcal{N}$ is given by

$$
\mathcal{N}=\left[N_{01}-N_{21}, N_{12}\right] .
$$

The terms involving $N_{1}, N_{2}, H_{1}$ and $H_{2}$ constitute the inputs to the two pendulum system. When there are no external forces

$$
H_{1}=H_{2}=0 \text {. }
$$

\section{APPENDIX C: PHYSICAL AND CONTROL PARAMETERS}

The definitions, symbols, and numerical values for the two rigid body system ${ }^{28}$ in the MKS system of units are given below. The rigid body parameters used in the simulation are

$$
\begin{aligned}
& m 1=4.6, m 2=7.6 \\
& R 1=[0,0,-0.267]^{\prime} \\
& R 2=[0,0,-0.247]^{\prime} \\
& S 2=[0,0,0.184]^{\prime} \\
& \text { The gravity constant and the vectors are } g=10 ; \\
& G r 1=m 1 * g *[0,0,-1]^{\prime} \\
& G r 2=m 2 * g *[0,0,-1]^{\prime}
\end{aligned}
$$

The moment of inertia of the two links are the two $3 \times 3$ matrices

$$
I 1=[j 01,0,0 ; 0, j 02,0 ; 0,0, j 03] \text {, and }
$$




$$
\begin{aligned}
I 2 & =[j 21,0,0 ; 0, j 22,0 ; 0,0, j 23] \\
K & r_{1}=100 I 1 \\
K r_{2} & =25 I 2 L r_{1}=0.2 K r_{1} \\
L r_{2} & =0.2 K r_{2} \\
K t_{1} & =25 m 1 I \text { den. } \\
L t_{1} & =0.2 K t_{1} \\
K t_{2} & =25 m 2 I \text { den. } \\
L t_{2} & =0.2 K t_{2} .
\end{aligned}
$$

The initial angles and angular velocities in the simulation are, for bodies 1 and 2, respectively,

$$
[0,0,0,0,10,0]^{\prime} \text { and }
$$$$
[0,0,0,0,-10,0]^{\prime} \text {. }
$$

\section{REFERENCES}

1. E. Whittaker, A treatise on the analytical dynamics of particles and rigid bodies, Dover Publications, New York, 1944.

2. J. Wittenberg, Dynamics of systems of rigid bodies, B.G. Teubner, Leipzig, 1977.

3. A. Shabana, Dynamics of multibody systems, Wiley, New York, 1989.

4. R. Featherstone and D. Orin, Robot dynamics: Equations and algorithms, in Proceedings of the 2000 International Conference on Robotics and Automation, San Francisco, CA, 2000, IEEE, New York, 2000, pp. 826834.

5. L. Nashner, C. Shupert, F. Horak, and F. Black, Organization of posture control: An analysis of sensory and mechanical constraints, Prog. Brain Res. 80 (1989), 411-418.

6. F. Horak and A. Kuo, Postural adaptation for altered environments, tasks and intentions, in Biomechanics and neural control of posture and movement, Springer, New York, 2000, pp. 267-281.

7. F. Horak and L. Nashner, Central programming of postural movements: Adaptation to altered supportsurface configurations, J. Neurophysiol. 55 (1986), 1369-1381.

8. D. Winter, F. Prince, C. Powell, and K. Zabjek, A unified theory regarding $\mathrm{a} / \mathrm{p}$ and $\mathrm{m} / \mathrm{i}$ balance during quiet stance, J. Neurophysiol. 75 (1996), 2334-2343.

9. H. Hemami and V.C. Jaswa, On a three-link model of the dynamics of standing up and sitting down, IEEE Trans. Syst. Man Cybern. 8 (1978), 115-120.

10. K. Barin, Evaluation of a generalized model of human postural dynamics and control in the sagittal plane, Biol. Cybern. 61 (1989), 37-50.

11. H. Hemami, A state space model for interconnected rigid bodies, IEEE Trans. Autom. Control 2 (1982), 376-382.

12. T.R. Kane and D.A. Levinson, Dynamics, theory and applications, McGraw-Hill, New York, 1985.

13. H. Hemami, A general framework for rigid body dynamics, stability and control, Trans. ASME, J. Dyn. Syst. Meas. 124 (2002), 241-251.

14. J. Craig, Introduction to robotics, mechanics and control, third ed., Prentice Hall, Englewood Cliffs, NJ, 1989.

15. H. Hemami and V. Utkin, On the dynamics and Lyapunov stability of constrained and embedded rigid bodies, Int. J. Control 6 (2002), 408-428.

16. H. Hemami and B. Dariush, Single rigid body representation, control and stability for robotic applications, in Proc. IRAC 2000, San Francisco, IEEE Robotic Society (2000), pp. 2340-2345.

17. B. Dariush, H. Hemami, and M. Parnianpour, Analysis and synthesis of human motion from external measurements, in Proc. IRAC 2000, San Francisco, IEEE Robotic Society (2000), pp. 4015-4020.

18. K. Barin, C.M. Seitz, and D. Welling, Effect of head orientation on the diagnostic sensitivity of posturography in patients with compensated unilateral lesions, Otolaryngol.-Head Neck Surg. 106 (1989), 355-362.

19. L. Ljung, System identification, theory for the user, Prentice Hall PTR, Englewood Cliffs, NJ, 1999.

20. G. Zames, Nonlinear operators for sysytem analysis, Tech. Rep. 370, Research Laboratory of Electronics, Mass. Inst. of Technology, Cambridge, MA, 1960.

21. D. George, Continuous nonlinear sysytems, Tech. Rep. 355, Research Laboratory of Electronics, Mass. Inst. of Technology, Cambridge, MA, 1959.

22. H. Hemami, B. Dariush, and K. Barin, Estimation of joint forces and torques in natural and artificial systems by nonlinear inverse dynamics, Tech. Rep. 55, The Ohio State University, Columbus, Ohio, 2002.

23. A. Isidori, Nonlinear control systems, Springer-Verlag, Berlin, 1989.

24. M. Krstic, I. Kanellakopoulos, and P. Kokotovic, Nonlinear adaptive control design, John Wiley and Sons, New York, 1995.

25. H. Khalil, Nonlinear systems, Prentice Hall, Englewood Cliffs, NJ, 1996.

26. H. Hemami, Towards a compact and computeradapted formulation of the dynamics and stability of multi rigid body systems, J. Automatic Control, University of Belgrade 1 (2002), 64-69.

27. P. Kokotovic, H.K. Khalil, and J. O'Reilly, Singular perturbation methods in control, Academic Press, New York, 1986.

28. H. Hemami and Y.-F. Zheng, Dynamics and control of motion on the ground and in the air with application to biped robots, J. Rob. Syst. 1 (1984), 101-116. 